\title{
The Biosulin equivalence in standard therapy (BEST) study - a multicentre, open-label, non-randomised, interventional, observational study in subjects using Biosulin 30/70 for the treatment of insulin-dependent type 1 and type 2 diabetes mellitus
}

\author{
D Segal, ${ }^{1} \mathrm{MB}$ BCh, FAAP, FACE; D Tupy, ${ }^{2} \mathrm{MD}$; L Distiller, ${ }^{3} \mathrm{BSc}, \mathrm{MB}$ BCh, FCP (SA), FRCP (London), FACE \\ ${ }^{1}$ Department of Paediatrics, Charlotte Maxeke Johannesburg Academic Hospital, Johannesburg, South Africa \\ ${ }^{2}$ Department of Internal Medicine, Hillbrow Community Hospital, Johannesburg, South Africa \\ ${ }^{3}$ Centre for Diabetes and Endocrinology, Johannesburg, South Africa
}

\section{Corresponding author: D Segal (david@endo.co.za)}

\begin{abstract}
Introduction. The need for more cost-effective insulin therapy is critical in reducing the burden on patients and health systems. Biosimilar insulins have the potential to dramatically lower healthcare costs by delivering insulin with a similar anti-glycaemic effect and adverse reaction profile.

Objectives. The purpose of this study was to confirm equivalence in glycaemic outcomes and side-effect profiles between Biosulin 30/70 and other human premixed insulin preparations on the South African market in a clinical practice setting.

Methods. Subjects in this interventional, observational, multicentre, open-label, prospective study were switched from their existing human premix insulin (Actraphane, Humulin 30/70 or Insuman) to the study insulin Biosulin 30/70. The primary endpoint was the change in HbAlc from baseline to 6 months.

Results. Seventy-seven adult patients with type $1(n=18)$ or type $2(n=59)$ diabetes were enrolled. The baseline HbA1c in the overall cohort was $7.9 \%, 8.0 \%$ at 3 months $(p=0.50)$ and $7.6 \%$ at 6 months $(p=0.14)$.There was a small increase in the total daily dose of insulin used in both the type 1 and type 2 cohort, from 0.62 to 0.65 units $/ \mathrm{kg} / \mathrm{day}(p=0.0004)$. There was no significant difference in weight in the study subjects during the 6-month period on Biosulin 30/70 ( $p=0.67$ ).

Conclusion. Biosulin 30/70 achieved at least equivalent glycaemic control to existing human premix insulins, with no reported new or severe adverse events. Increased use of biosimilar insulins has the potential for significant cost savings.
\end{abstract}

S Afr Med J 2013;103(7):458-460. DOI:10.7196/SAMJ.6345

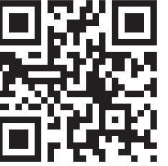

Diabetes accounted for at least \$US465 billion worth of health expenditure in $2011,11 \%$ of the total healthcare expenditure in the 20 - 79-year-old age range. ${ }^{[1]}$ According to the International Diabetes Federation (IDF), there are 1.9 million adults with diabetes in this age range in South Africa. ${ }^{[1]}$

The benefits of lifestyle change, exercise and weight loss are definite but difficult to achieve and maintain. Oral medications such as insulin sensitisers and insulin secretagogues are first- and secondline agents in the type 2 diabetes treatment armamentarium and are relatively inexpensive. Newer, more expensive agents (the DPP-IV inhibitors and GLP-1 analogues) have been added to the South African type 2 diabetes treatment algorithm and, although expensive, may further delay the introduction of insulin. ${ }^{[2]}$

Ultimately, however, progressive beta cell failure in patients with type 2 diabetes mellitus results in an ever-increasing proportion of patients requiring injected insulin to obtain and maintain glycaemic targets. Insulin remains the most effective glucose-lowering therapy. ${ }^{[2]}$ The addition of insulin dramatically increases the cost of management, with greater requirements for trained diabetes personnel, blood glucose test strips, injection devices, monitoring and the insulin itself.
Biosimilar insulins have the potential to dramatically lower healthcare costs by delivering insulin with similar anti-glycaemic effect and adverse reaction profile to standard, more expensive insulin preparations. ${ }^{[3]}$ The purpose of this study is to confirm equivalence in glycaemic outcomes and side-effect profiles between Biosulin 30/70 and other human premixed insulin preparations on the South African market in a clinical practice setting.

\section{Methods}

Seventy-seven subjects with type $1(n=18)$ and type 2 diabetes mellitus $(n=59)$ were enrolled in this interventional, observational, multicentre, open-label, prospective study.

Ethics approval was obtained from the Human Research Ethics Committee of the University of the Witwatersrand. Study subjects were recruited from the Hillbrow Community Hospital Diabetes Clinic (a state hospital facility) and two Centre for Diabetes and Endocrinology (CDE) centres in Johannesburg (private medical facilities). A real-world practice setting was used to gather data on the performance of the insulins across a range of economic and social strata in subjects with type 1 and type 2 diabetes.

Subjects who agreed to participate in the trial and after signing informed consent were enrolled and had anthropometrics measured. 
The subjects were then switched from their existing human premix insulin (Actraphane, Humulin 30/70 or Insuman) to the study insulin Biosulin 30/70 after education and demonstration on the new delivery device (the biopen). Most subjects were familiar with pen injection devices. Insulin dosages were kept unchanged at the first visit, but adjusted by the managing physician at subsequent 3-month visit according to their usual practice. No titration algorithm was in force. All oral hypoglycaemic agents and adjuvant medications were continued unchanged.

Blood was drawn at baseline, 3 months and 6 months for measurement of HbAlc. All samples were tested at the National Health Laboratory Services (NHLS) by a turbidimetric inhibition immunoassay (Cobas Integra 400) with a detection limit of $0.1 \mathrm{~g} / \mathrm{dl}$. The intra- and inter-assay coefficients of variation were $0.8 \%$ and $1.1 \%$ respectively. The change in $\mathrm{HbAlc}$ from baseline to 6 months was considered the primary endpoint of the study. All adverse events, and in particular the incidence and severity of any hypoglycaemic events, were recorded.

\section{Results}

Seventy-seven adult patients with type $1(n=18)$ or type $2(n=59)$ diabetes were enrolled at the 3 study centres. Thirty-six (47\%) of the 77 subjects were male. The type 1 cohort had a mean duration of diabetes of 7.2 years (range $1-21$ years) and the type 2 cohort had been requiring insulin for a mean of 7.4 years (range $1-21$ years). Patient characteristics are in Table 1. Subjects were grouped according to type of diabetes for better separation of subject characteristics but were pooled for the $\mathrm{HbAlc}$ data.

The baseline HbAlc in the overall cohort was $7.9 \%, 8.0 \%$ at 3 months $(p=0.50)$ and $7.6 \%$ at 6 months $(p=0.14)$. The type 1 cohort baseline HbAlc was $8.4 \%$ and improved to $8.0 \%$ at 6 months $(p=0.41)$. The type 2 cohort baseline HbAlc was $7.7 \%$, improving to $7.4 \%$ at 6 months $(p=0.19)$. There was no statistical difference in the primary endpoint of $\mathrm{HbAlc}$ after 6 months in the combined cohort or in the type 1 or type 2 cohorts on Biosulin 30/70 ( $p=0.14$ ) (Fig. 1).

There was a small but significant increase in the total daily dose of insulin used in both the type 1 and type 2 cohorts, from 0.62 to $0.65 \mathrm{u} / \mathrm{kg} /$ day $(p=0.0004)$. There was no significant difference in weight in the study subjects during the 6-month period on Biosulin $30 / 70$. The mean weight at baseline for the type 1 cohort was $68.1 \mathrm{~kg}$, increasing to $68.6 \mathrm{~kg}$ at study end $(p=0.52)$, and $91.2 \mathrm{~kg}$ declining to $90.8 \mathrm{~kg}(p=0.42)$ in the type 2 cohort. As expected, their BMIs were essentially unchanged $(p=0.74)$.

There were no severe hypoglycaemic episodes reported by the subjects in the 1 month prior to the study (on their pre-study insulin) and for the duration of the study on Biosulin 30/70. Most of the subjects were managed at the state hospital facility and did not perform self-monitored home blood glucose measurements.

\section{Discussion}

With the humanitarian and economic burdens of diabetes increasing and with the greatest demands and increases in developing countries, the need for more cost-effective insulin therapy is critical in reducing the financial burden on patients and health systems.

Biosimilars are distinctly different from generic drugs. Generic drugs are chemical entities that have an identical chemical composition to the parent drug and are relatively easy to manufacture. Typically, the delivered cost of a generic product is $10 \%$ to $70 \%$ that of the 'parent' drug.

Biosimilars, on the other hand, are protein compounds that rely on post-translational modification and folding for efficacy, and therefore are bio-equivalent rather than bio-identical. Because they are foreign proteins, and can elicit an antibody response that can attenuate their function or cause unwanted side-effects, biosimilars must follow precise manufacturing, processing and purification procedures, and are regulated to pass stringent laboratory and clinical trials before approval. Such biopharmaceuticals are made by only a handful of manufacturers using similar techniques involved in producing the parent product, and are therefore termed biosimilar. With many of the insulins reaching the end of their patents, these medications become attractive targets for production of biosimilar insulins. One of the obstacles to the adoption of biosimilar insulin is the notion that

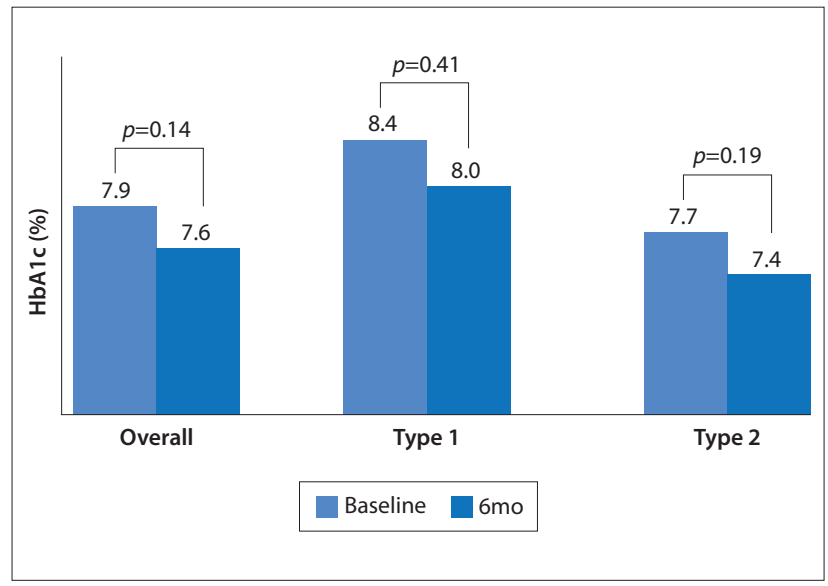

Fig. 1. HbA1c at baseline and after 6 months of therapy with Biosulin 30/70.

Table 1. Patient characteristics and data comparison

\begin{tabular}{|c|c|c|c|c|c|c|c|c|c|}
\hline & \multicolumn{3}{|l|}{ Total } & \multicolumn{3}{|l|}{ Type 1} & \multicolumn{3}{|l|}{ Type 2} \\
\hline$N$ & \multicolumn{3}{|l|}{77} & \multicolumn{3}{|l|}{18} & \multicolumn{3}{|l|}{59} \\
\hline Sex (male/female) & \multicolumn{3}{|l|}{$36 / 41$} & \multicolumn{3}{|l|}{$7 / 11$} & \multicolumn{3}{|l|}{$29 / 30$} \\
\hline Age (years), mean (range) & \multicolumn{3}{|c|}{$49.7(26-75)$} & \multicolumn{3}{|c|}{$38.9(26-60)$} & \multicolumn{3}{|c|}{$52.1(26-75)$} \\
\hline \multirow{2}{*}{$\begin{array}{l}\text { Duration of insulin use } \\
\text { (years), mean }\end{array}$} & \multicolumn{3}{|l|}{7.3} & \multicolumn{3}{|l|}{7.2} & \multicolumn{3}{|l|}{7.4} \\
\hline & Baseline & 6 months & $p$-value & Baseline & 6 months & $p$-value & Baseline & 6 months & $p$-value \\
\hline $\mathrm{TDD}(\mathrm{u} / \mathrm{kg} /$ day $)$ mean $\pm \mathrm{SD}$ & $0.62 \pm 0.19$ & $0.65 \pm 0.20$ & 0.0004 & $0.71 \pm 0.19$ & $0.76 \pm 0.18$ & 0.02 & $0.59 \pm 0.19$ & $0.61 \pm 0.17$ & 0.01 \\
\hline Weight (kg), mean \pm SD & $85.3 \pm 17.2$ & $85.1 \pm 16.9$ & 0.67 & $68.1 \pm 9.6$ & $68.6 \pm 9.5$ & 0.52 & $91.2 \pm 15.2$ & $90.8 \pm 15.1$ & 0.42 \\
\hline $\mathrm{BMI}\left(\mathrm{kg} / \mathrm{m}^{2}\right)$, mean $\pm \mathrm{SD}$ & $31.1 \pm 6.0$ & $31.1 \pm 5.9$ & 0.74 & $25.9 \pm 4.1$ & $26.1 \pm 4.0$ & 0.55 & $32.9 \pm 5.5$ & $32.8 \pm 5.5$ & 0.48 \\
\hline HbAlc $(\%)$, mean \pm SD & $7.9 \pm 1.6$ & $7.6 \pm 1.7$ & 0.14 & $8.4 \pm 1.6$ & $8.0 \pm 1.8$ & 0.41 & $7.7 \pm 1.5$ & $7.4 \pm 1.7$ & 0.19 \\
\hline
\end{tabular}


it may be less effective. Some of the concerns have been legitimate, with a number of biosimilar insulins failing to meet stringent registration standards. ${ }^{[4]}$ However, ongoing vigilance and extensive clinical trials will continue to be required to ensure the safety and efficacy of new biosimilar insulins.

Biosulin 30/70 L, N and R were approved for use in South Africa by the Medicines Control Council (MCC) in 2005. To achieve this, Biosulin trials by Pharmovs in South Africa demonstrated to the MCC that Biosulin has the same clinical properties as the parent insulin already on the market, and additionally that it did not have any unexpected and new adverse effects in diabetics.

In this non-randomised, interventional 6-month study comparing glycaemic control of existing human insulin mixes (Actraphane, Humulin 30/70 and Insuman) with Biosulin 30/70, Biosulin 30/70 achieved at least equivalent glycaemic control with no reported new or severe adverse events. This surveillance study of Biosulin 30/70 in a real-world medical practice setting expands the safety and efficacy data on record.

Despite the more complex production process and regulatory requirements for biosimilars, they offer a price reduction of between $35 \%$ and $50 \%$ of the parent insulin and analogue insulins. ${ }^{[5]}$

A meta-analysis to assess the effects of short-acting analogues in patients with type 2 diabetes failed to find any difference in HbAlc between regular human insulin and short-acting analogues. ${ }^{[5]}$ There was, however, a small benefit in favour of short-acting analogue insulins in reducing the frequency of severe hypoglycaemic episodes from a median of 1.4 episodes per 100 patient years to 0.3 episodes. ${ }^{[5]}$ Horvath et al. ${ }^{[6]}$ compared long-acting analogues Detemir and Lantus with NPH insulin in adult patients with type 2 diabetes and also found no Alc advantage. The efficacy of these analogue insulins in type 1 diabetes is more established although the actual advantages are minimal. ${ }^{[7]}$

Overall, more expensive analogue insulins offer minimal advantages over regular insulin in the management of type 2 diabetes, and increased use of biosimilar insulins has the potential for significant cost savings with no loss in patients' glycaemic outcomes. There is no doubt that biosimilar insulins such as Biosulin will play an ever-increasing role in the management of diabetes.

Acknowledgements. This study was funded through an educational grant supplied by Bioswiss.

\section{References}

1. International Diabetes Federation. IDF Diabetes Atlas 2012;c2003. http://www.idf.org/diabetesatlas (accessed 9 July 2012)

. Amod A, Motala A, Levitt N, et al. The 2012 SEMDSA treatment algorithm for type 2 diabetes. EMDSA 2012;17(1):S36-S40.

. Home P. Biosimilar insulins. Diabetes Voice 2011;56(2):40-43.

4. Kuhlmann M, Marre M. Lessons learned from biosimilar epoetins and insulins. Brit J Diab Vasc Dis 2010;10(2):90-97.

5. Siebenhofer A, Plank J, Berghold A, et al. Short acting insulin analogues versus regular human insulin in patients with diabetes mellitus. Cochrane Database Syst Rev 2006(2):CD003287.

6. Horvath $\mathrm{K}$, Jeitler K, Berghold A, et al. Long-acting insulin analogues versus NPH insulin (human isophane insulin) for type 2 diabetes mellitus. Cochrane Database Syst Rev 2007(2):CD005613.

7. Distiller LA, Joffe BI. From the coalface: does glargine insulin improve hypoglycaemic episodes, . Distiller LA, Joffe BI. From the coalface: does glargine insulin improve hypoglycaemic episodes,
glycaemic control or affect body mass in type 1 diabetic subjects who are attending a 'routine' diabetes glycaemic control or affect body mass in type
clinic? Diabetologia 2006;49(11):2793-2794.

Accepted 2 November 2012. 